\title{
CMB polarimetry with BICEP: instrument characterization, calibration, and performance
}

Yuki D. Takahashi, Denis Barkats, John O. Battle, Evan M. Bierman, James J. Bock, et al.

Yuki D. Takahashi, Denis Barkats, John O. Battle, Evan M. Bierman, James J. Bock, H. Cynthia Chiang, C. Darren Dowell, Eric F. Hivon, William L. Holzapfel, Viktor V. Hristov, William C. Jones, J. P. Kaufman, Brian G. Keating, John M. Kovac, Chao-Lin Kuo, Andrew E. Lange, Erik M. Leitch, Peter V. Mason, Tomotake Matsumura, Hien T. Nguyen, Nicolas Ponthieu, Graca M. Rocha, Ki Won Yoon, P. Ade, L. Duband, "CMB polarimetry with BICEP: instrument characterization, calibration, and performance," Proc. SPIE 7020, Millimeter and Submillimeter Detectors and Instrumentation for Astronomy IV, 70201D (19 July 2008); doi: 10.1117/12.790306

Event: SPIE Astronomical Telescopes + Instrumentation, 2008, Marseille, France 


\title{
CMB polarimetry with BICEP: instrument characterization, calibration, and performance
}

\author{
Yuki D. Takahashi*a, Denis Barkats ${ }^{b}$, John O. Battle ${ }^{c}$, Evan M. Bierman ${ }^{d}$, James J. Bock ${ }^{b, c}$, \\ H. Cynthia Chiang ${ }^{b}$, C. Darren Dowell ${ }^{c}$, Eric F. Hivon ${ }^{e}$, William L. Holzapfel $^{a}$, \\ Viktor V. Hristov ${ }^{b}$, William C. Jones ${ }^{b}$, J. P. Kaufman ${ }^{d}$, Brian G. Keating ${ }^{d}$, John M. Kovac ${ }^{b}$, \\ Chao-Lin $\mathrm{Kuo}^{f}$, Andrew E. Lange ${ }^{b}$, Erik M. Leitch ${ }^{c}$, Peter V. Mason ${ }^{b}$, Tomotake Matsumura ${ }^{b}$, \\ Hien T. Nguyen ${ }^{c}$, Nicolas Ponthieu ${ }^{g}$, Graca M. Rocha ${ }^{b}$, Ki Won Yoon ${ }^{h}$, P. Ade ${ }^{i}$, L. Duband ${ }^{j}$ \\ ${ }^{a}$ University of California, Berkeley, USA; \\ ${ }^{b}$ California Institute of Technology, Pasadena, USA; \\ ${ }^{c}$ Jet Propulsion Laboratory, Pasadena, USA; \\ ${ }^{d}$ University of California, San Diego, USA; \\ ${ }^{e}$ Institut d'Astrophysique de Paris, France; \\ ${ }^{f}$ Stanford University, Palo Alto, USA; \\ $g$ Universite Paris XI, Orsay, France; \\ ${ }^{h}$ National Institute of Standards and Technology, Boulder, USA; \\ ${ }^{i}$ Cardiff University, UK; \\ ${ }^{j}$ Commissariat à l'Énergie Atomique, Grenoble, France;
}

\begin{abstract}
BICEP is a ground-based millimeter-wave bolometric array designed to target the primordial gravity wave signature on the B-mode polarization of the cosmic microwave background (CMB) at degree angular scales. Currently in its third year of operation at the South Pole, BICEP is measuring the CMB polarization with unprecedented sensitivity at 100 and $150 \mathrm{GHz}$ in the cleanest available $2 \%$ of the sky, as well as deriving independent constraints on the diffuse polarized foregrounds with select observations on and off the Galactic plane. Instrument calibrations are discussed in the context of rigorous control of systematic errors, and the performance during the first two years of the experiment is reviewed.
\end{abstract}

Keywords: cosmic microwave background polarization, mm-wave, bolometers, cosmology, inflation, South Pole

\section{INTRODUCTION}

The clearest evidence of an inflationary origin of the Big Bang would be a detection of a curl-mode polarization ("B-mode") in the CMB arising from gravitational wave perturbations. The B-mode polarization is expected to peak at degree angular scales, with the magnitude of the power spectrum described by the ratio $r$ of the initial tensor to scalar perturbation amplitudes, a quantity directly related to the energy scale of inflation. The current upper limit is $r<0.2$ from CMB temperature measurement at large angular scales by WMAP, combined with constraints from the Type Ia supernovae and the baryon acoustic oscillations. ${ }^{1}$ More directly with the B-mode polarization, upper limits of $\sim 0.8 \mu \mathrm{K}$ rms has been placed by WMAP at multipole of $\ell \sim 65$ and by QUAD at $\ell \sim 200$, respectively. ${ }^{2}$ These limits are still well above the expected levels of confusion from the Galactic synchrotron and dust polarization foregrounds in the cleanest regions of the sky or from lensing which converts the much brighter gradient-modes ("E-modes") to B-modes at smaller angular scales. An instrument designed to target the expected peak of the gravity-wave signature at $\ell \sim 100$, with judicious selection of observed field and exquisite control of systematic errors, should be able to probe deeply below the current upper limit on $r$. Utilizing proven bolometric technologies albeit with unprecedented total sensitivity, BICEP is just such an effort. Probing down to $r=0.1$, which corresponds to a signal at degree scales of $\sim 0.1 \mu \mathrm{K} \mathrm{rms}$, requires careful instrument characterization and calibration to minimize systematic contamination in the polarization measurement.

\footnotetext{
*yuki@ bolo.berkeley.edu; phone 1510642 4359; fax 15106435204
} 


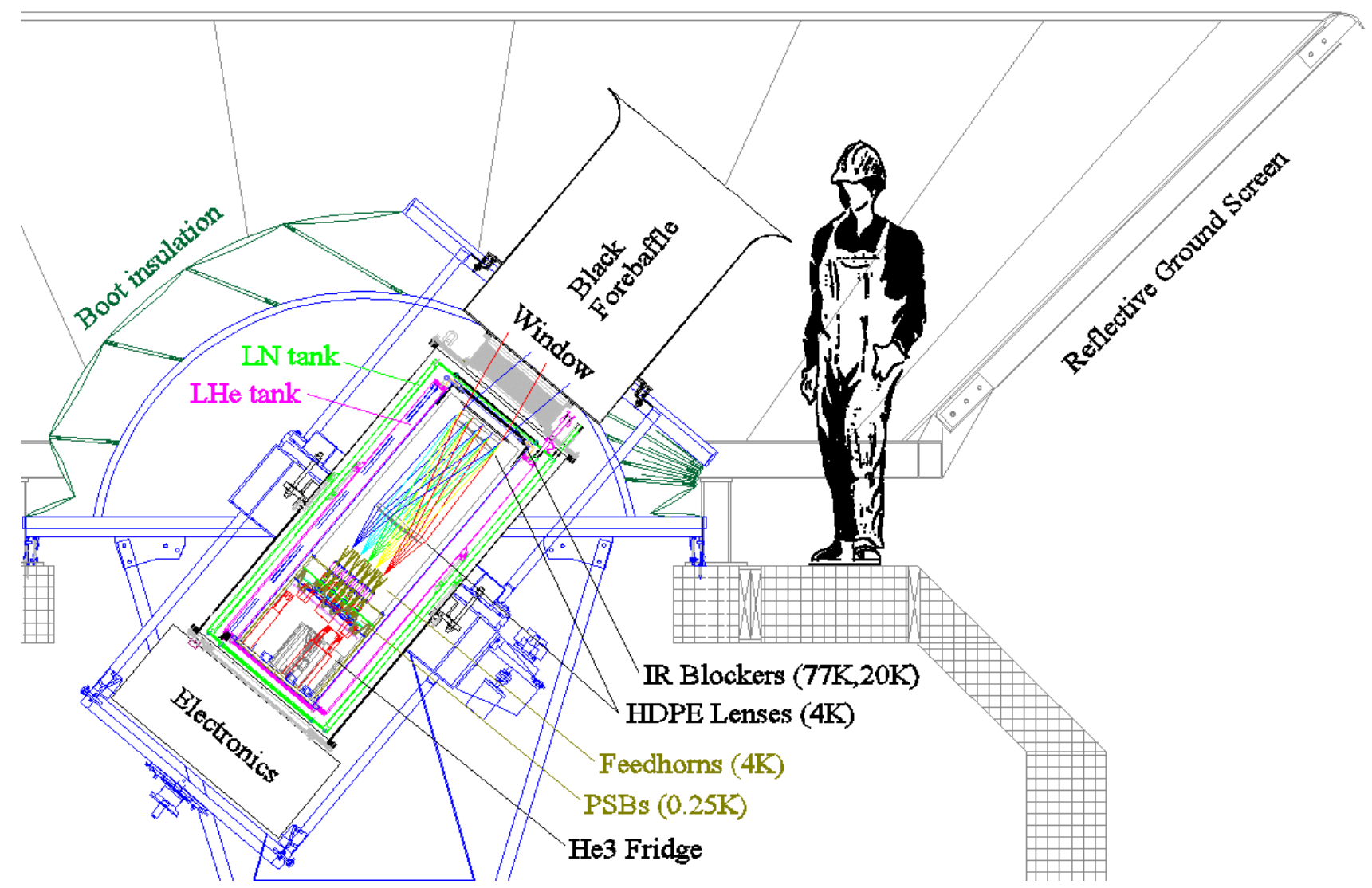

Figure 1. BiCEP telescope at the South Pole, at its lowest observing elevation of $50^{\circ}$. The $\mathrm{LN}_{2} / \mathrm{LHe}_{\text {torroidal }}$ cryostat encloses the entire $4 \mathrm{~K}$ telescope, and houses a ${ }^{4} \mathrm{He} /{ }^{3} \mathrm{He} /{ }^{3} \mathrm{He}$ sorption refrigerator that cools the bolometers to $250 \mathrm{mK}$.

\section{OVERVIEW OF INSTRUMENT AND OBSERVING STRATEGY}

Installed at the South Pole in November 2005, BICEP is a compact refractor with 49 pairs of polarization sensitive bolometers (PSBs) at primarily $100 \mathrm{GHz}$ and $150 \mathrm{GHz}$ with $0.9^{\circ}$ and $0.6^{\circ}$ beams, respectively. The bolometers use neutron transmutation doped (NTD) Germanium thermistors to sense the optical power incident on the absorber mesh. Figure 1 shows an overall picture of the instrument as set up in a building 800 meters from the geographic pole at 2800-meter altitude. The instrument design and the observation strategy were described in Yoon et al. ${ }^{3}$ (hereafter referred to as the "2006 Paper").

Figure 2 shows the number of detector pairs employed in each observing year, as well as the locations, orientations, and nominal full width half maximum (FWHM) of all the beams. Between the first and second years, we added $220 \mathrm{GHz}$ feedhorns in place of two of the $150 \mathrm{GHz}$ ones along with the appropriate filters. We also replaced four bolometers because of their slow temporal response, high noise level, or poor polarization efficiency. We have kept BICEP cold and running without any interruption since this minor upgrade in December 2006.

With this instrument, we map an $800 \mathrm{deg}^{2}$ field daily by scanning a $60^{\circ}$ range in azimuth at $2.8^{\circ} / \mathrm{s}$ with hourly $0.25^{\circ}$ steps in elevation. Each two-day observing cycle (Figure 3) begins with 6 hours allocated for cycling the refrigerator, filling liquid nitrogen (every 2 days) and liquid helium (every 4 days), measuring mount tilts, and performing star pointing calibrations. The scan speed was selected to provide sufficient signal modulation against the $1 / f$ atmospheric drifts while limiting motion-induced thermal fluctuations at the detectors in our science frequency band to sub-nK levels. 


\begin{tabular}{|l|cc|}
\hline & 2006 & $2007 / 2008$ \\
\hline $100 \mathrm{GHz}$ & $25(19)$ & $25(22)$ \\
$150 \mathrm{GHz}$ & $24(14)$ & $22(15)$ \\
$220 \mathrm{GHz}$ & 0 & 2 \\
dark bolometers & 6 & 6 \\
thermistors (NTD) & 6 & 8 \\
resistors $(5 \mathrm{M} \Omega)$ & 6 & 5 \\
\hline
\end{tabular}

Figure 2. Above: The number of detector pairs for each observing year. (In parenthesis is the number of pairs used in the current CMB analysis.) In addition to bolometers behind feedhorns, along the perimeter of the focal plane are "dark" bolometers not coupled to radiation, un-etched modules used as thermistors, and fixed resistors for diagnostic purpose.

Right: Nominal 2007/2008 layout of the BICEP beams, with six sections having alternating "Q" or "U" detector orientations with respect to the radial vector from the center. The observations are performed with the focal plane orientations of $-45^{\circ}, 0^{\circ}, 135^{\circ}$, and $180^{\circ}$, providing two independent and complete $\mathrm{Q} / \mathrm{U}$ coverages of the field.

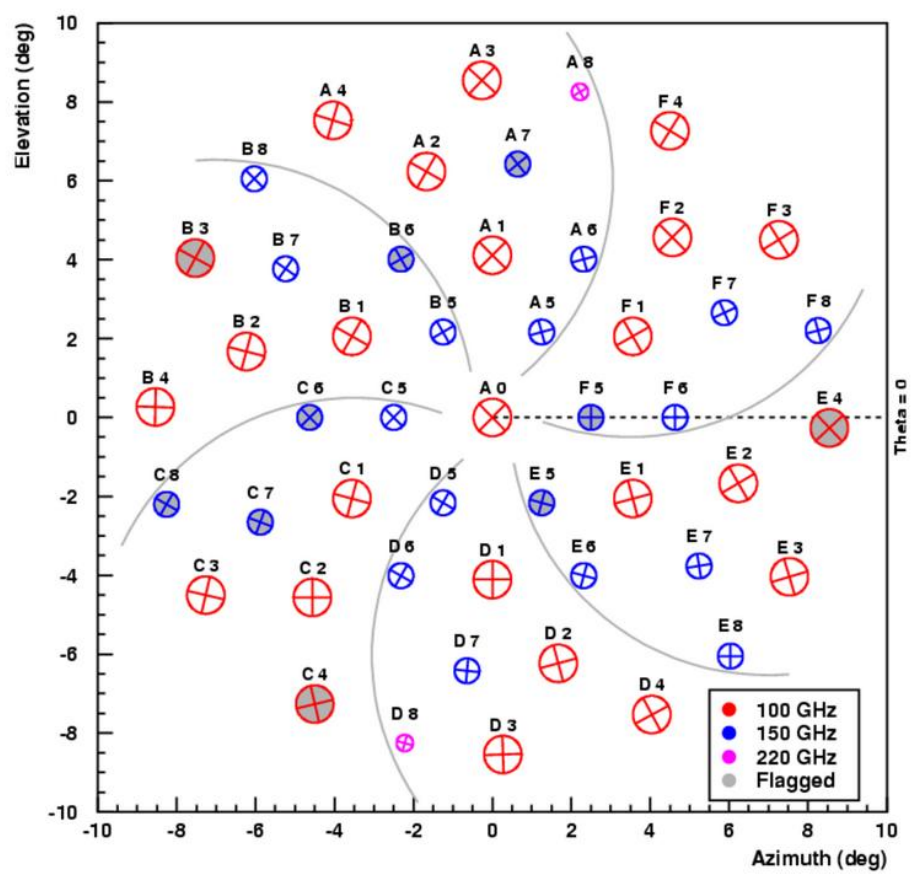

BICEP 2-Day Observing Cycle

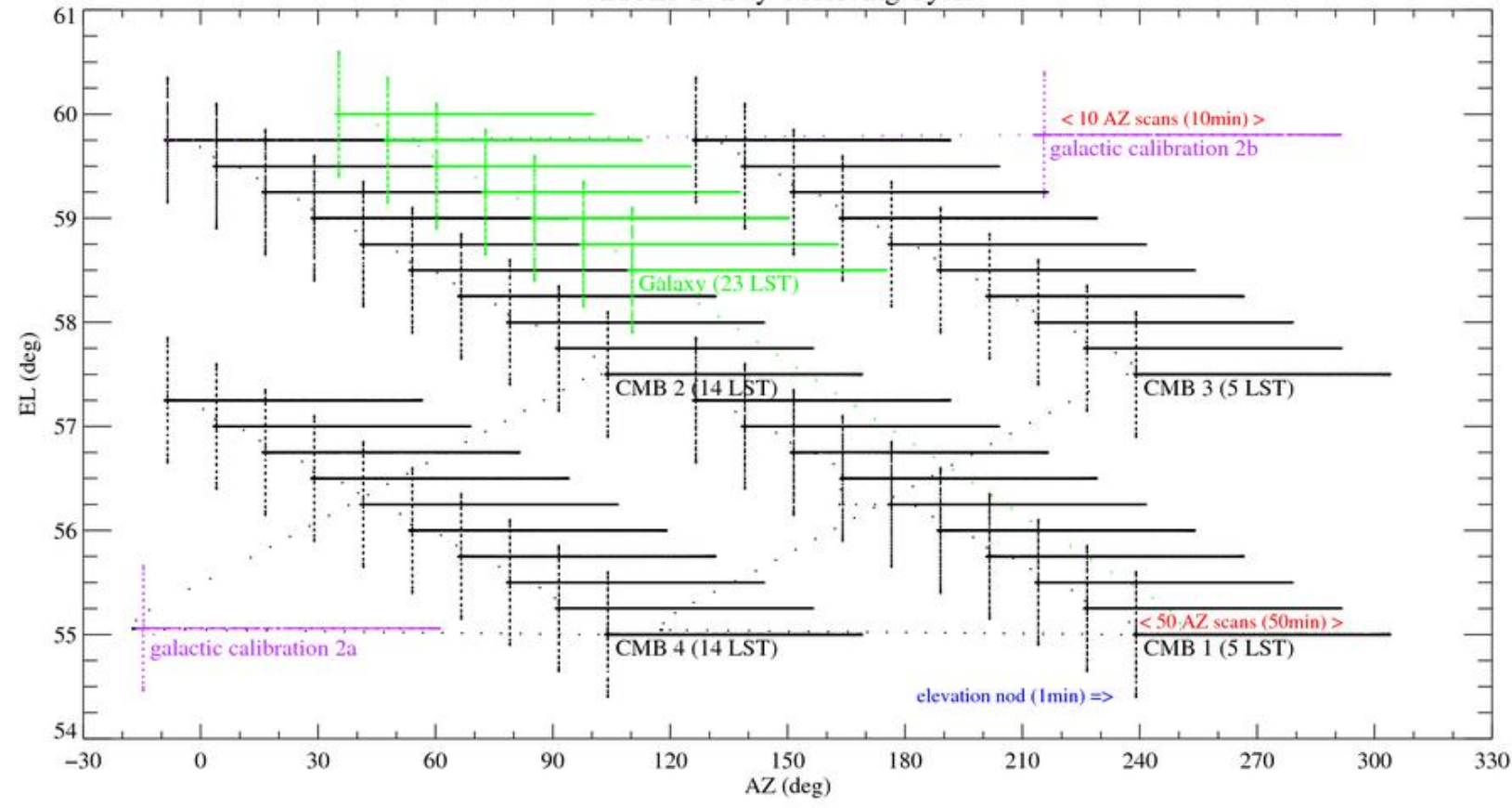

Figure 3. A 48-hour observing cycle consists of 6 hours for cycling the refrigerator (not shown), two 9-hour blocks of raster scans on our main CMB field on the first day (CMB 1 and CMB 2), a 6-hour block of similar raster scans on the Galaxy, and two more 9-hour blocks on the main CMB field on the second day (CMB 3 and CMB 4), performed over the same azimuth range as on the first day but in the alternate coverage order of the elevation range, allowing a test for ground contamination. At the beginning and the end of each set of 50 -minute azimuth scans, a $\pm 0.6^{\circ}$ elevation "nod" is performed to measure relative gains of every bolometer. 


\section{INSTRUMENT CHARACTERIZATION}

To process the collected time streams into co-added polarization maps with systematic errors tolerable for our target sensitivity, a very accurate characterization of the detectors and their beams is crucial. The voltage response of a PSB to Stokes parameters $I, Q, U$ can be modeled as:

$$
d(t)=H_{t} \otimes \frac{s}{2} \int \mathrm{d} \nu A_{e} F_{\nu}^{\star} \int \mathrm{d} \Omega P(\Omega)\left[I+\frac{1-\epsilon}{1+\epsilon}(Q \cos 2 \psi+U \sin 2 \psi)\right]
$$

where $\psi$ is the orientation angle of the PSB's maximum response to linearly polarized light, $\epsilon$ is the polarization leakage, $P(\Omega)$ is the beam function, $F_{\nu}^{\star}$ is the spectral response, $A_{e}$ is the effective antenna area, $s$ is the responsivity at $0 \mathrm{~Hz}$, and $H_{t} \otimes$ signifies the convolution of the time-domain impulse response associated with the detector's frequency transfer function.

The requirement for the accuracy in the specification of these instrumental quantities were guided by simulated observations of the CMB sky to quantify the systematic error that would induce false B-mode signal at the level of $r=0.1$. This benchmark level corresponds to B-mode fluctuations of $\sim 0.1 \mu \mathrm{K} \mathrm{rms} \mathrm{at} \mathrm{degree} \mathrm{scales.}$ Table 1 summarizes the instrument properties and $r=0.1$ benchmark levels for their characterization, as well as the measured results described in this section. Each instrument property has been characterized to a level of precision more than adequate for B-mode measurements at the $r=0.1$ level.

Table 1. Potential Systematic Errors for BiCEP.

\begin{tabular}{|c|c|c|}
\hline Instrument properties & Benchmark $(r=0.1)$ & Measured \\
\hline Relative gain error: $\Delta\left(s_{1}-s_{2}\right) / s$ & $1.0 \%$ & $0.4 \%$ \\
\hline Differential beam size $^{a}:\left(\sigma_{1}-\sigma_{2}\right) / \bar{\sigma}$ & $4.0 \%$ & $<0.2 \%$ \\
\hline Differential pointing $^{a}:\left|\overrightarrow{r_{1}}-\overrightarrow{r_{2}}\right| / \bar{\sigma}$ & $1.5 \%$ & $1.3 \%^{b}$ \\
\hline Differential ellipticity: $\left(e_{1}-e_{2}\right) / 2$ & $9.0 \%$ & $<0.1 \%$ \\
\hline Polarization orientation error: $\Delta \psi$ & $8^{\circ}$ & $0.7^{\circ}$ \\
\hline Polarized sidelobes to Galaxy ${ }^{c}$ & $-8 \mathrm{dBi}$ & $<-38 \mathrm{dBi}$ \\
\hline Polarized sidelobes to ground ${ }^{c}$ & $-19 \mathrm{dBi}$ & $<-38 \mathrm{dBi}$ \\
\hline Cold-stage temperature stability ${ }^{d}: \Delta \mathrm{T}$ & $1.3 \mathrm{nK}$ & $<0.5 \mathrm{nK}$ \\
\hline Optics temperature stability ${ }^{d}: \Delta \mathrm{T}_{R J}$ & $10 \mu \mathrm{K}$ & $<0.7 \mu \mathrm{K}$ \\
\hline
\end{tabular}

\subsection{Detector temporal transfer function}

Analysis of the time ordered data from each detector begins by deconvolving the temporal response using the measured frequency-domain optical transfer function of the detector. Since the transfer function is proportional to the gain of the detector at each frequency, it directly affects the relative gains of a PSB pair to be differenced. The relative transfer functions must thus be measured to well within the $1 \%$ benchmark desired for the relative gain.

At the nominal scan speed of $2.8^{\circ} / \mathrm{s}$ in azimuth at $\sim 60^{\circ}$ elevations, our target angular scales of $\ell=30-300$ fall into the frequency band of approximately $0.1-1 \mathrm{~Hz}$. Since the elevation nods described in Section 4.1 probe the relative gains at $\sim 0.02 \mathrm{~Hz}$, the transfer functions were measured down to $0.01 \mathrm{~Hz}$.

The primary measurement technique involved analyzing the step response to a fast-switching square-wave source (Gunn oscillator or broadband noise source) operating at $0.01-0.1 \mathrm{~Hz}$, while under optical loading conditions representative of CMB observations (Figure 4). Possible dependency on background loading and detector non-linearity were explored by repeating the measurement with weakly emissive foam pieces in the beam and/or with different signal strengths. 
Figure 5 shows the measured transfer functions for a representative PSB pair. The relative gain mismatch due to measurement errors is found to be $<0.3 \%$ over the frequency range of $0.01-1 \mathrm{~Hz}$. Although the measured transfer functions fit the following model

$$
\tilde{H}(\omega) \propto \frac{1-\alpha}{\left(1-i \omega \tau_{1}\right)\left(1-i \omega \tau_{2}\right)}+\frac{\alpha}{\left(1-i \omega \tau_{\alpha}\right)},
$$

their signal-to-noise ratio was high enough that they could be directly inverse Fourier transformed to define the deconvolution kernels. The median time constants were $\tau_{1} \sim 20 \mathrm{~ms}$ and $\tau_{2} \sim 5 \mathrm{~ms}$. From the first year, 6 channels at $150 \mathrm{GHz}$ were excluded from CMB analysis due to excessive low frequency roll off (worst combinations of $\alpha>0.05$ and $\tau_{\alpha}>300 \mathrm{~ms}$ ). Two of the worst were in a PSB pair and were replaced at the end of the year.

Between the two years, the transfer function measurements generally agreed to within $0.5 \% \mathrm{rms}$ across the signal band. Two exceptions were excluded from the first year CMB data, since the second year measurements were more reliable at the lowest frequencies. Details of the measurements and analysis are in Yoon $2007 .^{4}$

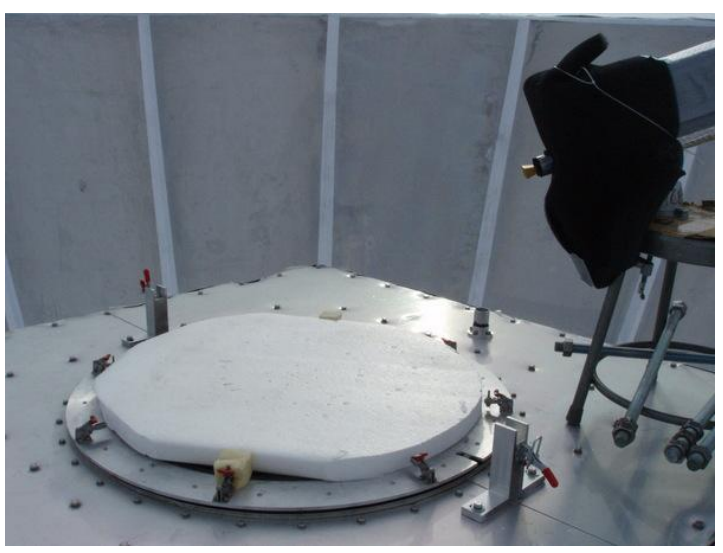

Figure 4. A setup in front of the telescope aperture for measurement of transfer functions. Metal washers are embedded in the transparent zotefoam sheet to scatter the PIN switched signal into the beam while keeping the total loading similar to that during nominal CMB observations.
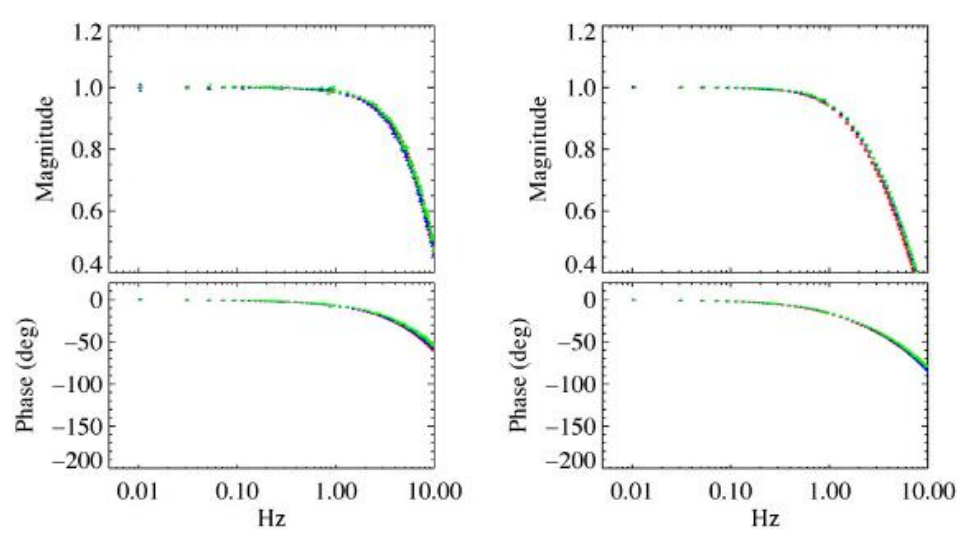

Figure 5. Measured transfer functions with error bars for a typical pair of PSBs under different loading conditions and signal magnitudes: low-loading/high-signal (red), low-loading/low-signal (purple), high-loading/high-signal (blue), and high-loading/low-signal (green).

\subsection{Beam widths and pointings}

Ideal differencing of a PSB pair is limited by any mismatch in the beams that can turn unpolarized signal into false polarization. The difference between two nearly circular beams can be categorized into three dominant types: differential size, pointing, and ellipticity. The beam size and ellipticity differences are sensitive to the second derivative of the temperature field, while differential pointing is also sensitive to the temperature gradient. If the direction and magnitude of differential pointing can be established, the resulting leakage of the CMB temperature gradients into polarization can in principle be estimated and accounted for.

The beams were mapped by raster scanning a bright source at various telescope orientations about the boresight. The far field of the telescope is about 50 meters from the aperture, which permitted measurements in a high bay prior to telescope deployment and on rooftops at the South Pole. In the high bay, a thermal blackbody source was used at a 40-meter distance, consisting of a liquid nitrogen load behind chopper blades covered with ambient temperature absorber. At the South Pole, a 30-foot fold-over mast was installed on the rooftop outside of the ground screen, allowing us to position a source at $60^{\circ}$ elevation 10 meters away. For a truly far-field measurement, an additional mast was installed on the roof of another building, at a distance of nearly 200 meters, and a flat mirror was temporarily mounted above the telescope to direct the beams down to the low elevation of the mast as well as to low elevation astronomical sources (Figure 6). The sources used included an ambient temperature chopper against the cold sky, a broadband noise source, the Moon, and Jupiter. 


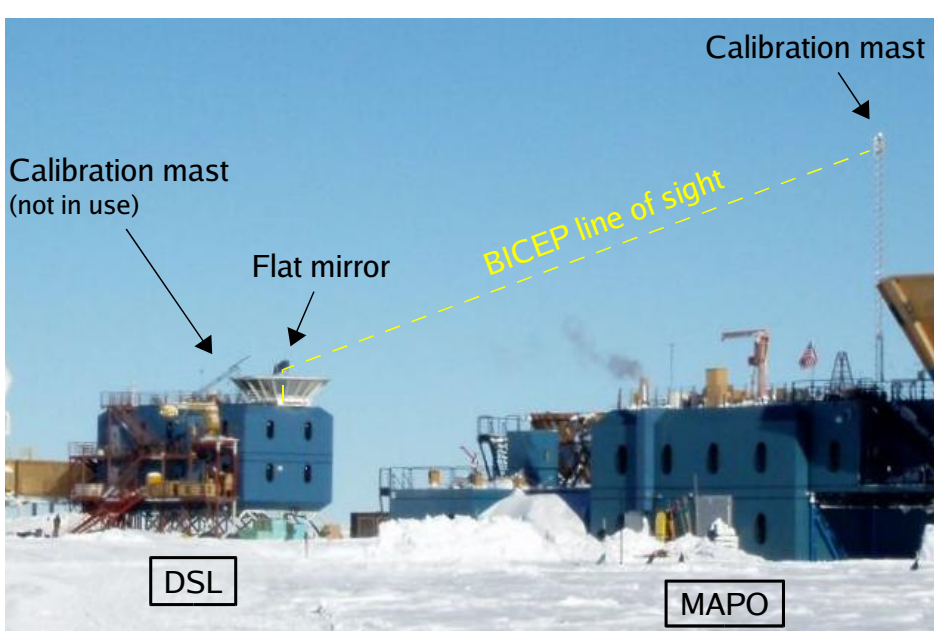

Figure 6. The beam mapping setup on site consisted of sources Figure 7. Beams for each PSB pair are normalized mounted on top of fold-over masts. When using the mast on the and differenced to produce this composite differential MAPO building (200 m from the Dark Sector Laboratory), a flat beam map. The overplotted lines show the fitted cenmirror is mounted to direct the beams over the ground screen.

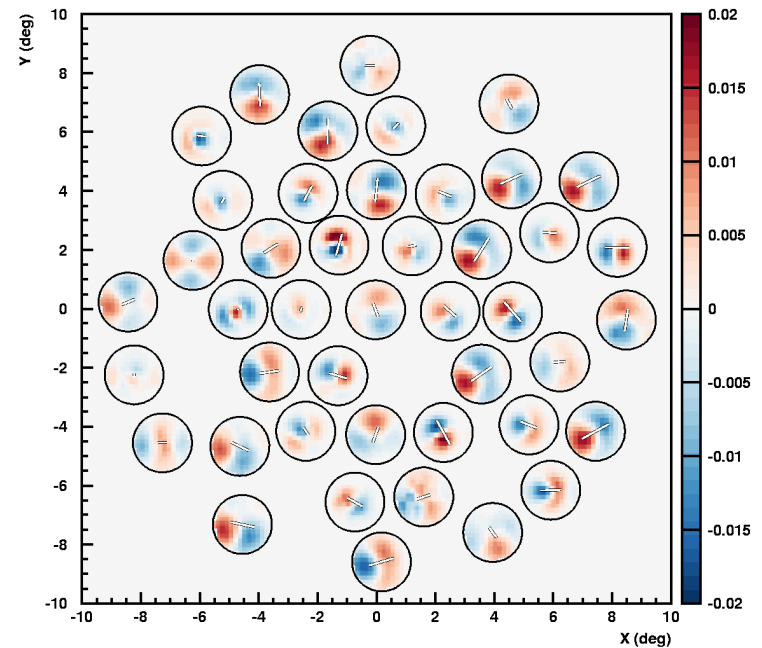

troid offsets magnified by a factor of 100 .

The measured beams fit the Gaussian model with typically $1 \%$ residuals in amplitude. The fitted centroids are repeatable to about $0.02^{\circ}$, although the accuracy of the absolute locations is currently limited by uncertainties in parallax corrections. The average measured FWHMs are $0.93^{\circ}$ and $0.60^{\circ}$ for 100 and $150 \mathrm{GHz}$, respectively, about $5 \%$ smaller than predicted from physical optics simulations. The beams have small ellipticities of $<1 \%$ for $100 \mathrm{GHz}$ and $<1.5 \%$ for $150 \mathrm{GHz}$.

A composite of the differenced normalized beams for each PSB pair is shown in Figure 7, measured with the broadband noise source, an amplified thermal source that is ideal for probing low-level effects. The largest beam mismatch effect is a pointing offset that gives rise to dipole patterns in many of the differenced beams. The median differential pointing offset is $0.004^{\circ}$, or $\sim 1 \%$ of the beam size $\bar{\sigma}$, and the offsets were repeatable between observations performed at different telescope orientations of both the noise source and the Moon to within the measurement uncertainty, $0.4 \%$ of $\bar{\sigma}$. The measurements of differential size and ellipticity are less repeatable, but the upper limits of $0.2 \%$ and $0.1 \%$, respectively, are negligibly small.

Finally, to co-add maps made with different PSB pairs, the actual locations of all the beams must be determined. This was accomplished by first making a full season co-added map of the CMB using the design locations and then cross-correlating the temperature anisotropy pattern with single detector maps to adjust the individual beam coordinates. These adjusted coordinates were then used to iterate this process, resulting in derivation of the absolute beam locations with an uncertainty of $0.03^{\circ} \mathrm{rms}$, based on the agreement between the first and second years. For BICEP, using the CMB temperature fluctuations proved more effective than attempting a similar procedure with Eta Carinae, the brightest compact source accessible.

\subsection{Polarization orientations and efficiencies}

Angles of the PSBs can vary from their design orientations based on the tolerances with which they were mounted. The deviation from perfect orthogonality of a pair simply reduces its efficiency for polarization; however, an error in the overall orientation of the pair can lead to mixing of E-modes into B-modes. To ensure this mixing is well below the level of the B-mode spectrum for $r=0.1$, the calibration procedure was designed to determine the polarization orientations to within $\pm 1^{\circ}$.

Another factor, though less important, is that the PSBs are not perfectly insensitive to polarization components orthogonal to their orientations, effectively reducing the polarization efficiency to $1-\epsilon$. To achieve $10 \%$ accuracy in the amplitudes of the polarization power spectra, our goal was to measure polarization leakages $\epsilon$ to within \pm 0.02 . 
As described in the 2006 Paper, the polarization orientations were measured using a rotatable dielectric sheet device shown in Figure 8. The measurements were performed several times throughout each observing year, and produced repeatable results for the individual PSB orientations with $0.3^{\circ} \mathrm{rms}$. The PSB pairs were orthogonal to within $0.1^{\circ}$, and together were within $\pm 1^{\circ}$ of the design orientations shown in Figure 2 . The absolute orientation was found to within $\pm 0.7^{\circ}$, limited by the accuracy in determining the dielectric sheet orientation relative to the azimuthal orientation of the entire array.

This absolute orientation was confirmed by two other independent methods that were used to measure the polarization leakages. One method employed a rotatable wire grid on top of the telescope aperture, with a chopper modulating the completely polarized load between the ambient absorber and the cold sky (Figure 9). Fitting a sinusoidal curve to the PSB response as a function of the wire grid angle gives the polarization efficiency and orientation. The measurements for both years gave polarization leakages within \pm 0.02 of each other, with a median value of $\epsilon=0.05$.

The other method used a modulated broadband noise source with a rectangular horn behind a wire grid, mounted on the mast 200 meters away (Figure 10). This source was raster scanned by each of our beams with 18 different detector orientations with respect to the wire grid, fitting a 2-dimensional Gaussian to each raster. The measured leakages were slightly lower with a median of $\epsilon=0.04$, and the uncertainty was \pm 0.01 based on the scatter against the results from the first method. One $150 \mathrm{GHz}$ bolometer was flagged for having an $\epsilon>0.12$ and was replaced at the end of the first year.

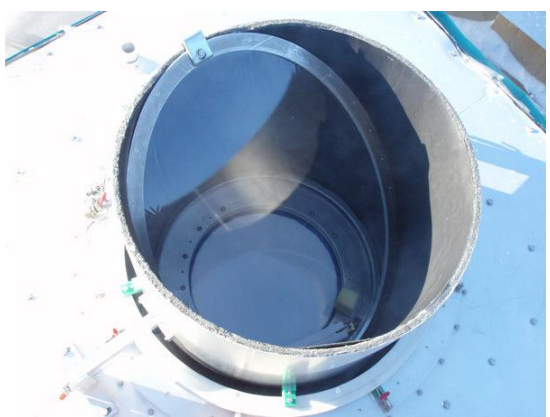

Figure 8. The dielectric sheet calibrator, consisting of a beam-filling polypropylene sheet and a highly emissive black lining as an ambient load, injects partially polarized radiation into the telescope aperture below. The device is mounted on the azimuth stage, which can rotate about the telescope's boresight when pointed at zenith.

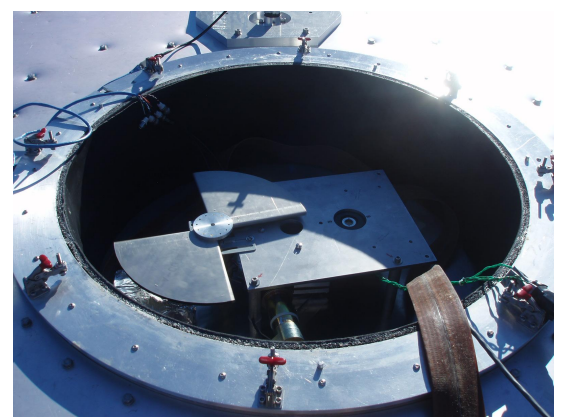

Figure 9. A device above the cryostat window for measuring $\epsilon$ and $\psi$. The window is covered with a metal plate with a 2-cm Eccosorb aperture, and a 10 -cm diameter wire grid is on a rotation stage under the circular aperture of the rectangular plate. The chopper modulates the load between the ambient temperature and the cold sky.

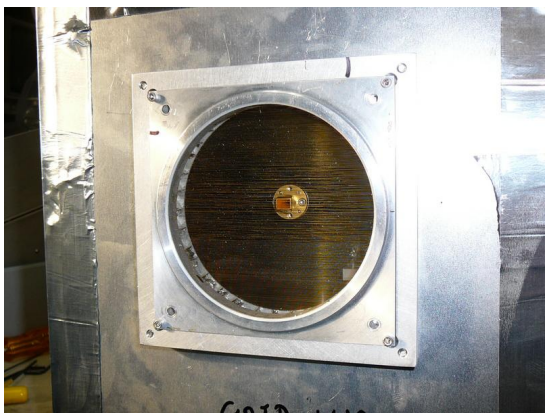

Figure 10. Another calibration source used on top of a mast for measuring $\epsilon$ and $\psi$. The broadband noise source at 100 or $150 \mathrm{GHz}$ outputs power through the rectangular feedhorn oriented for either vertical or horizontal polarization and through a precisely aligned wire grid to minimize cross-polarized signal.

\subsection{Spectral response}

As described in Section 4.1, relative gain calibration for differencing out the unpolarized component of the CMB is based on elevation nods that subject the bolometers to varied atmospheric loads. Because the CMB and the atmospheric emission have different spectral shapes, the spectral response of the PSB pair must match well enough to prevent errors in the relative responsivity to the CMB.

The spectral response of each channel was measured using polarized Fourier Transform Spectrometers with a maximum resolution of $0.3 \mathrm{GHz}$. Within each frequency band, the spectra were very similar from channel to channel (average spectra shown in Figure 11), and the preliminary upper limit on the expected differential gains due to the spectral mismatch appears to be at an acceptable level.

In addition to the main band, we verified that there are no significant responses at higher frequencies due to leaks in the low-pass filter stacks. Thick grill filters with cut-off frequencies of $165 \mathrm{GHz}$ and $255 \mathrm{GHz}$ were used at the telescope aperture one at a time and the response to a chopped thermal source was measured. $150 \mathrm{GHz}$ 
channels showed no sign of leaks down to the noise floor at $-35 \mathrm{~dB}$, while $100 \mathrm{GHz}$ channels exhibited leaks at $\sim-25 \mathrm{~dB}$ level somewhere beyond $255 \mathrm{GHz}$. The magnitude of this small $(\sim 0.3 \%)$ leak was consistent between the PSBs in each pair, so that possible effect on relative responsivities is expected to be negligible.

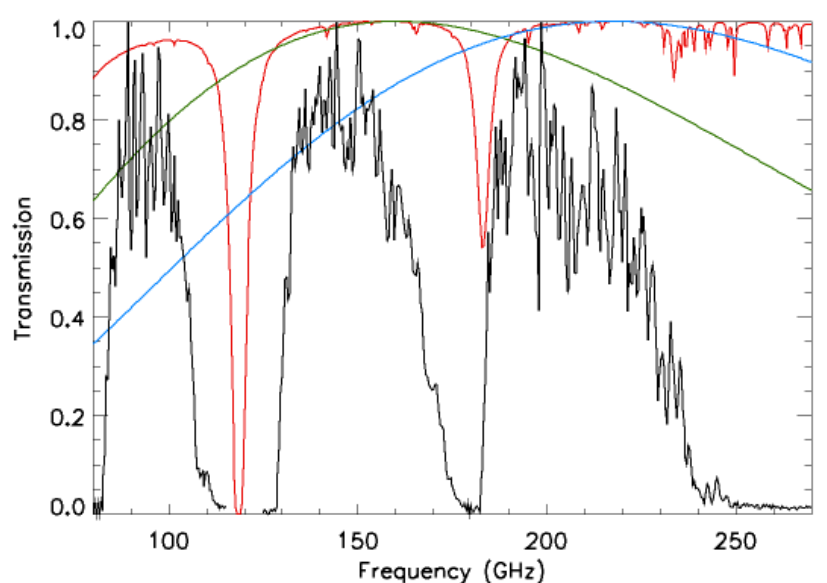

Figure 11. The average measured spectral response for each of BICEP's frequency bands, normalized with respect to the maximum. Overplotted are the atmospheric transmission at the South Pole (red), the CMB spectrum (green) and its temperature derivative (blue).

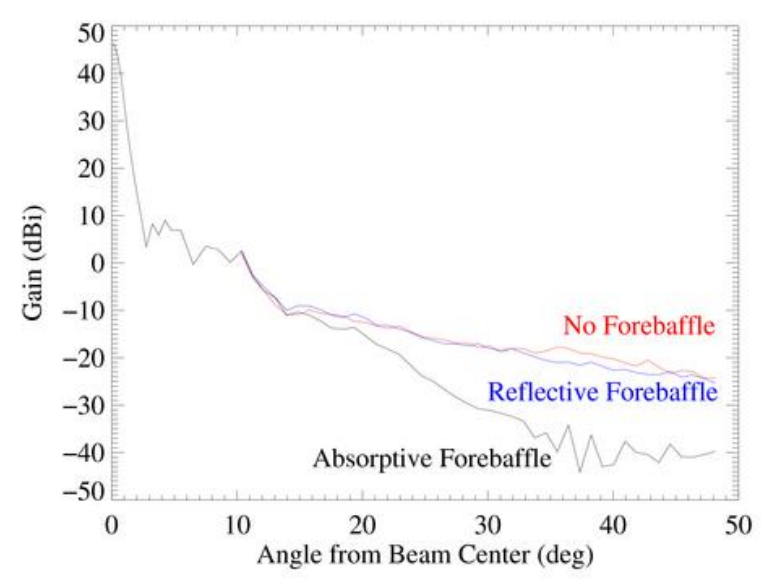

Figure 12. The far sidelobe response for the central feed. Being cylindrical, a forebaffle with a bare aluminum surface had little effect on the sidelobe rejection, while the addition of the absorptive lining (Eccosorb HR-10) provided up to an additional $15 \mathrm{~dB}$ attenuation. When the telescope is at its lowest elevation of $50^{\circ}$, the lip of the outer ground screen is $\sim 30^{\circ}$ from the beam center of the central feed.

\subsection{Far sidelobe rejection}

The design criterion for the ground shields was to reject the ground radiation to the level below our target B-mode polarization sensitivity. Polarized sidelobes can produce spurious signals by also coupling to emissions from the Galaxy and the outer ground screen.

The far sidelobe response of BICEP including the forebaffle (see Figure 1) was measured using a modulated microwave source (Gunn oscillator or broadband noise source) on top of the mast within a line of sight. The telescope was stepped away in elevation up to $60^{\circ}$ away from the source in $0.5^{\circ}$ increments, making one revolution about the boresight at each step for a complete azimuthal coverage. This measurement was performed with several source attenuations down to $-50 \mathrm{~dB}$ to probe the many decades of gain with sufficient signal-to-noise ratio while also measuring the main beam without saturating the detector.

The sidelobe maps are azimuthally averaged to obtain a radial profile (Figure 12). To evaluate the far sidelobe rejection performance, this level of response with a measured upper limit of $20 \%$ polarization was convolved with a model map of the predicted dust emission from the Galaxy. The resulting B-mode contamination in our CMB field was found to be negligible. The same exercise was repeated for a conservative model of varying snow accumulation on the ground screen panels. The contamination was larger, but still comfortably suppressed by the high rejection levels achieved (see Table 1). Potential ground contamination can also be directly probed in our data through jackknife tests. If necessary, our scan strategy allows us to separate it from the sky signal, although so far no ground signal subtraction has proven necessary.

\section{CALIBRATION}

In addition to relatively static instrumental properties that are characterized at most annually, possibly dynamic quantities are calibrated routinely throughout the observing season. These include the detector responsivities, especially the relative gains of the PSB pairs, and the telescope pointing parameters. 


\subsection{Relative detector gains}

Polarization measurement with BICEP relies on PSB pair differencing, in which the relative gains within each pair must be accurately determined to prevent the bright unpolarized component from inducing false polarized signals. Simulations suggest that the pair relative gains should be accurate to at least $1 \%$ to limit the leakage of CMB temperature anisotropy into significant B-mode contamination.

Relative gains are derived from elevation nods performed once at the beginning and once at the end of every one-hour constant-elevation scan set (Figure 3). The elevation motion of the telescope during a nod is a rounded triangle wave with a peak-to-peak amplitude of $1.2^{\circ}$ that injects a $\sim \pm 0.1 \mathrm{~K}$ optical loading signal (Figure 13). The bolometer responses are fit to a simple air mass model of atmospheric loading versus elevation $\left(T_{a t m} \propto\right.$ $1 / \sin (E L))$ to derive the relative gains across the array.

The elevation motion generates thermal disturbances on the focal plane, although they are limited by performing the nod slowly over a 50 second period. To reduce the effect of the thermally-induced false signals, the two elevation nods for each scan set are performed in opposite patterns (up-down-return and down-up-return) and the average gain is used. While the two patterns result in a small systematic difference in the individual gains, the pair relative gains are consistent to within $\pm 0.3 \%$ with no systematic difference between the two patterns. Over a time scale of months, the measured relative gains are stable with $\sim 1 \%$ rms and exhibit no systematic variation with the optical loading level. As a cross check, relative gains have also been derived from correlating time-stream atmospheric fluctuations within PSB pairs, and the results agree with the elevation nods within $\pm 0.3 \%$.

The common-mode rejection was ultimately assessed by cross-correlating individual PSB pair-sum and pairdifference maps to quantify the level of leakage of the CMB temperature anisotropy into pair-differences. The median correlation over the PSB pairs was $0.4 \%$ over the angular scales of interest, validating the differencing technique to this level.

The infrared flash calibrator described in the 2006 Paper gave a very repeatable $(0.2 \%$ rms $)$ response between the beginning and the end of the one-hour scan sets and also showed that the individual gains are stable with $1 \% \mathrm{rms}$ across the full elevation range. However, there are several uncertainties associated with the calibrator, including $\sim 3 \mathrm{~K}$ excess optical loading introduced by the swing arm, and unknown polarization of the infrared source. The relative gains from the flash calibrator have therefore not been used in the analysis.

Assuming that the average responsivity to the $\mathrm{CMB}$ of all the detectors is constant, the measured individual gains are scaled for each 1-hour scan set such that the mean gain over each frequency band is unity. The gain-adjusted time streams are co-added to form CMB maps in detector voltage units, and the BICEP and WMAP temperature maps (identically smoothed and filtered) are cross-correlated to derive absolute gains for each of BICEP's frequency bands. The absolute gains are consistent with those obtained from the dielectric sheet calibrator, which have $10 \%$ uncertainty.

\subsection{Telescope pointing}

Pointing errors greater than $1 \%$ of the beam size $\bar{\sigma}$ could contaminate polarization at the $r \sim 10^{-4}$ level. ${ }^{5}$ Although this effect is far below our current sensitivity target, our star pointing camera (described in the 2006 Paper) was developed to achieve this $1 \%$ accuracy, which for BICEP corresponds to $10^{\prime \prime}$.

The telescope pointing is established using star observations from an optical camera rigidly bolted to the top surface of the cryostat. Pointing calibrations are performed every two days during the refrigerator cycles, weather permitting, as well as before and after each mount re-leveling. In each calibration run, the telescope points at 24 stars (down to magnitude 3 ) at boresight orientation angles of $-45^{\circ}, 45^{\circ}$, and $135^{\circ}$, and the azimuth and elevation offsets required to center the stars are recorded. The pointing data are fit to a 10-parameter model with typical residuals of $10-12^{\prime \prime}$ rms. The pointing model has been checked by cross-correlating the CMB temperature anisotropy patterns between the pointing-corrected daily maps and the cumulative map; no systematic offsets or drifts were detected.

The tilt of the telescope mount is monitored every two days with two orthogonal tilt meters mounted on the azimuth stage. We have observed seasonal tilt changes of up to $0.5^{\prime}$ per month, possibly due to the building settling on the snow, and generally have re-leveled the mount before the tilt exceeds $1^{\prime}$. 


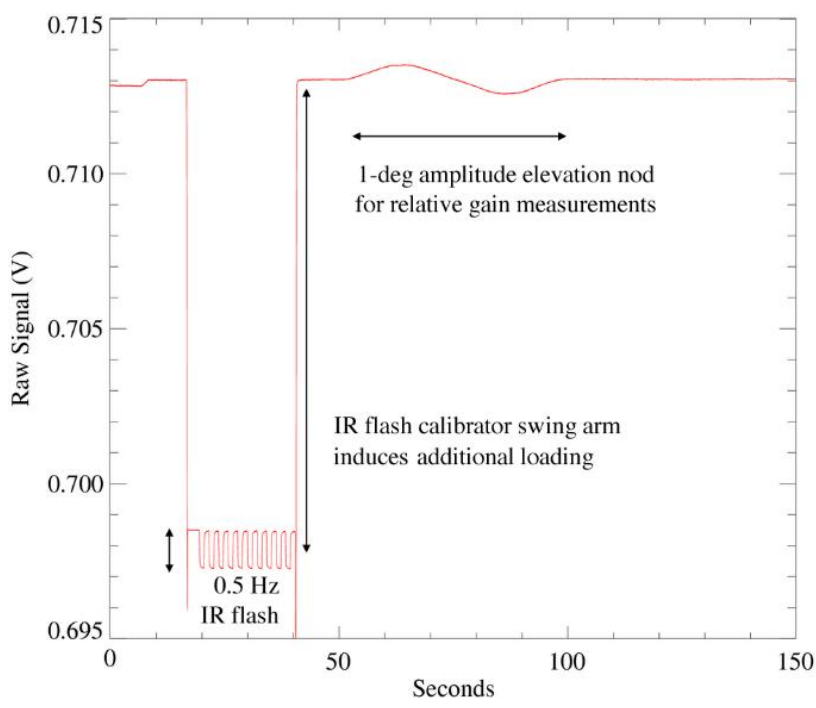

Figure 13. A bolometer time stream during a relative responsivity calibration procedure. An infrared source is swung into the beam to inject a signal of very stable amplitude, useful for tracking any gain variations. The elevation nod uses the atmospheric loading variation.
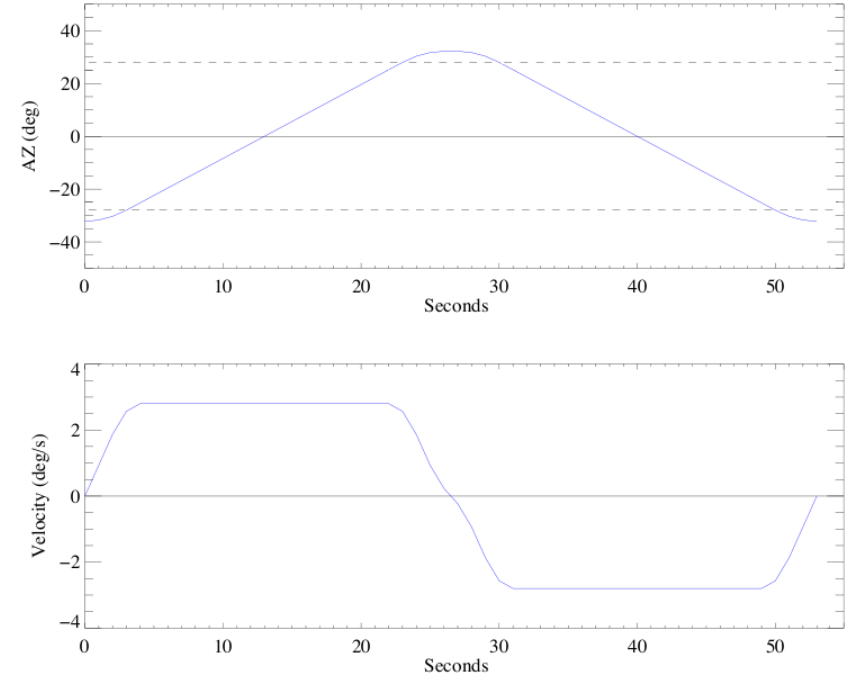

Figure 14 . A $2.8^{\circ} / \mathrm{s}$ azimuth scan profile used in mapping the CMB field. The acceleration during turn-arounds is limited to $1.4^{\circ} / \mathrm{s}^{2}$; the jerk is limited to $0.47^{\circ} / \mathrm{s}^{3}$. A 20 second portion of mostly constant-velocity scan is used from each direction, resulting in a $75 \%$ scan efficiency.

\section{PERFORMANCE}

We began our telescope installation work at the South Pole in November 2005, cooled down the cryostat in December, and captured first astronomical light a month later. Following calibration measurements and tests of the observing strategy, BICEP began CMB observations in February 2006. The instrument has since operated nearly continuously and will continue observing in its current configuration until the end of 2008 .

\subsection{Observing efficiency}

Excluding any incomplete 9-hour blocks, BICEP acquired 180 days of CMB observations during 2006, a feat previously unmatched by any other telescope at the South Pole operating in its first year. A significant fraction of the first year was devoted to calibration measurements, and the amount of CMB data taken in 2007 increased to 245 days. CMB data acquired during the Austral summer are generally of lower quality than the winter data because of several factors, including mediocre weather conditions and increased station activities. Although BICEP is capable of observing during the summer - there is no evidence for Sun contamination - we restrict our CMB analysis to data taken during February-November.

The first 2.5 months of data in 2006 were excluded from the current analysis because different scan strategies were used at the time, and a small but measurable level of radio frequency (RF) interference was detected in the warm receiver electronics. The RF interference was successfully eliminated by carefully sealing the RF shield surrounding the electronics. As a coarse weather cut, we have excluded 9-hour blocks if the relative gains derived from elevation nods have a standard deviation above $20 \%$, averaged over the channels. After these cuts, 116 days in 2006 and 226 days in 2007 remain for our baseline CMB analysis.

The data set is slightly reduced due to the occasional presence of snow on the window. Infrared images of the telescope window were captured every hour, and $7 \%$ of the scan sets in 2006 and $2 \%$ in 2007 were flagged for visible snow accumulation. Furthermore, $3 \%$ of PSB pair time streams are flagged due to cosmic ray hits, glitches, and $>3 \%$ relative gain drifts over one-hour periods. Accounting for the $75 \%$ scan efficiency (Figure 14) and the scheduled calibration routines, the net CMB observing efficiency is $60 \%$ during the CMB observing blocks and $45 \%$ overall during each two-day cycle. 


\subsection{Thermal stability}

Temperature drifts in the focal plane and optics can produce artifacts if they have a scan-synchronous component. The refrigerator cools to $233-236 \mathrm{mK}$, and the temperature of the focal plane is stabilized at approximately $250 \mathrm{mK}$ using a $100 \mathrm{k} \Omega$ resistor as a control heater (nominally depositing $\sim 0.1 \mu \mathrm{W}$ ) in a PID feedback loop with a sensitive thermistor. During the first year, we used a thermistor on the focal plane closest to the thermal strap connected to the refrigerator. Since thermal disturbances presumably transmit through the strap, our control scheme for the subsequent years used two new pairs of thermistors mounted on the strap itself, next to two redundant control heaters. Along with additional reinforcement of the thermal straps using Vespel supports and an increased response speed of the control loop, the spectra of the focal plane thermistors improved visibly from the first year to the second.

The temperature stability of the focal plane varies with azimuth scan speed and the telescope orientation about its boresight, most likely due to microphonically induced heating. The stability was investigated under a variety of telescope operating conditions, including scan speeds in a range of $1.0^{\circ} / \mathrm{s}-4.0^{\circ} / \mathrm{s}$, and 16 evenly spaced boresight orientation angles. We selected our $2.8^{\circ} \mathrm{s}$ operating scan speed and four boresight angles $\left\{-45^{\circ}, 0^{\circ}, 135^{\circ}, 180^{\circ}\right\}$ by minimizing the variance of the scan-synchronous thermistor signals.

The bolometers' responsivities to the bath temperature were measured by fitting the output voltage to a $10 \mathrm{mK}$ drop at the end of each refrigerator cycle. The median thermal responsivity is $0.8 \mu \mathrm{K}_{\mathrm{CMB}} / \mathrm{nK}_{\mathrm{FP}}$, and the median mismatch within PSB pairs is $0.08 \mu \mathrm{K}_{\mathrm{CMB}} / \mathrm{nK}_{\mathrm{FP}}$. In order to meet the $r=0.1$ target at $\ell \sim 100$, thermal instabilities in the focal plane must be controlled to better than $1.3 \mathrm{nK} \mathrm{rms}$. The measured level of thermal fluctuations in BICEP is $0.5 \mathrm{nK} \mathrm{rms}$ and flat over the frequency range corresponding to $\ell=30-300$.

Since emission from BICEP's optics is expected to be unpolarized, the main concern with optics temperature drifts is in mis-calibration of PSB pair optical relative gains, which are controlled to better than 1\%. Scansynchronous fluctuations averaged over all bolometers and over a 2 month period showed only $0.7 \mu \mathrm{K}_{R J}$ variation, integrated over the relevant frequency range.

\subsection{Preliminary Results}

Temperature and polarization maps from BICEP's first two years of operation, as well as jackknives formed by subtracting maps made at the two frequencies, are shown in Figure 15. The CMB temperature anisotropy has been measured with high signal-to-noise, as demonstrated by the absence of structure in the temperature jackknife map. The faint striping in the latter, caused by residual atmospheric noise, is successfully removed by PSB differencing.

The E- and B-mode maps are calculated from apodized Stokes Q/U maps and are Wiener-filtered according to the expected E-mode signal divided by the beam function. Degree-scale E-mode structure is resolved at the map level, while the E and B jackknife maps and B signal maps are consistent with noise. The jackknife maps show no evidence of foreground contamination.

After 3700 hours of total integration time, the noise per $1 \mathrm{deg}^{2}$ pixel in the $\mathrm{Q}$ and $\mathrm{U}$ jackknife maps is measured to be $0.78 \mu \mathrm{K} \mathrm{rms}$ for $100 \mathrm{GHz}$ and $0.62 \mu \mathrm{K} \mathrm{rms}$ for $150 \mathrm{GHz}$, which is consistent with expectations. The map noise levels translate to a preliminary estimate of noise equivalent temperature (NET) per detector of $560 \mu \mathrm{K} \sqrt{s}$ at $100 \mathrm{GHz}$ and $430 \mu \mathrm{K} \sqrt{s}$ at $150 \mathrm{GHz}$.

\section{ACKNOWLEDGMENTS}

BICEP has been possible through support from NSF Grant No. OPP-0230438, Caltech President's Discovery Fund, Caltech President's Fund PF-471, JPL Research and Technology Fund, and the late J. Robinson. BGK gratefully acknowledges support from NSF PECASE Award \#AST-0548262. We thank the South Pole Station staff for their continuing support and Steffen Richter for being a winter over we can rely on. We thank our colleagues in Acbar, Boomerang, QUaD, Bolocam, and Spt for the advice and helpful discussions, and Kathy Deniston for logistical and administrative support. 

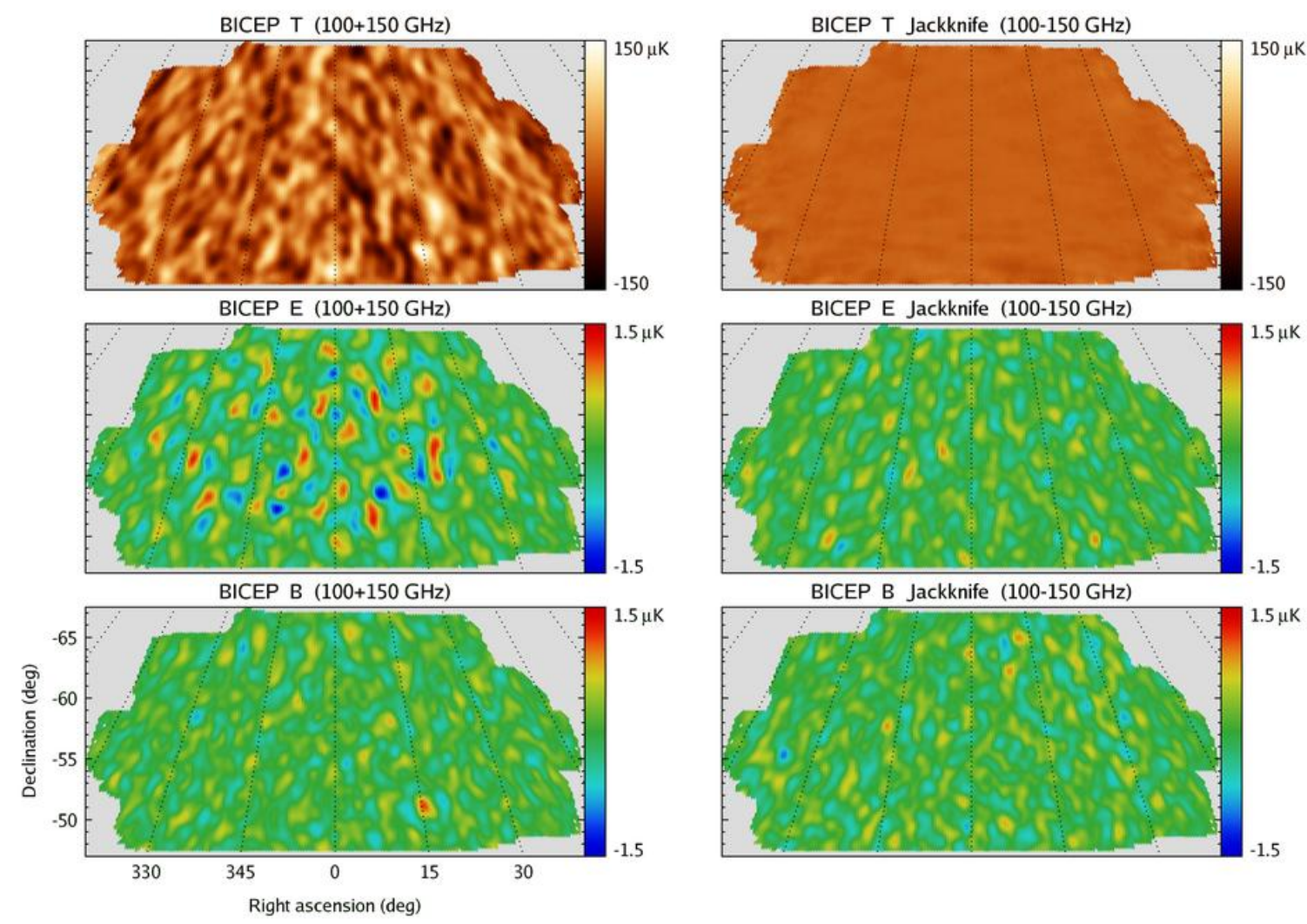

Figure 15. Maps of the first two years of data, including jackknives (right column). A 3rd-order polynomial is removed from each half-scan, and the maps are smoothed to $1^{\circ}$ resolution. Top row: CMB temperature anisotropy. The faint striping in the jackknife map is due to residual atmospheric noise, which is removed by PSB differencing. Bottom rows: Stokes Q and U maps are combined to produce Wiener-filtered E- and B-mode polarization maps. The E signal map shows degree-scale structure at the expected level, while the other three maps are consistent with noise.

\section{REFERENCES}

[1] Komatsu, E. et al., "Five-Year Wilkinson Microwave Anisotropy Probe (WMAP) Observations: Cosmological Interpretation," ArXiv e-prints $\mathbf{8 0 3}$ (Mar. 2008).

[2] QUaD collaboration: J. Hinderks, Ade, P., Bock, J., Bowden, M., Brown, M. L., Cahill, G., Carlstrom, J. E., Castro, P. G., Church, S., Culverhouse, T., Friedman, R., Ganga, K., Gear, W. K., Gupta, S., Harris, J., Haynes, V., Kovac, J., Kirby, E., Lange, A. E., Leitch, E., Mallie, O. E., Melhuish, S., Murphy, A., Orlando, A., Schwarz, R., O' Sullivan, C., Piccirillo, L., Pryke, C., Rajguru, N., Rusholme, B., Taylor, A. N., Thompson, K. L., Tucker, C., Wu, E. Y. S., and Zemcov, M., "QUaD: A High-Resolution Cosmic Microwave Background Polarimeter," ArXiv e-prints 805 (May 2008).

[3] Yoon, K. W., Ade, P. A. R., Barkats, D., Battle, J. O., Bierman, E. M., Bock, J. J., Brevik, J. A., Chiang, H. C., Crites, A., Dowell, C. D., Duband, L., Griffin, G. S., Hivon, E. F., Holzapfel, W. L., Hristov, V. V., Keating, B. G., Kovac, J. M., Kuo, C. L., Lange, A. E., Leitch, E. M., Mason, P. V., Nguyen, H. T., Ponthieu, N., Takahashi, Y. D., Renbarger, T., Weintraub, L. C., and Woolsey, D., "The Robinson Gravitational Wave Background Telescope (BICEP): a bolometric large angular scale CMB polarimeter," in [Millimeter and Submillimeter Detectors and Instrumentation for Astronomy III], Zmuidzinas, J., ed., Proc. SPIE 6275, $62751 \mathrm{~K}(2006)$.

[4] Yoon, K. W., Design and deployment of BICEP: A novel small-aperture CMB polarimeter to test inflationary cosmology, PhD thesis, California Institute of Technology (2007).

[5] Hu, W., Hedman, M. M., and Zaldarriaga, M., "Benchmark parameters for CMB polarization experiments," Phys. Rev. D 67, 043004 (Feb. 2003). 